\title{
Antiretroviral prophylaxis for the prevention of HIV infection: future implementation challenges
}

"The concept of PrEP is not new; it builds on the premise that effective therapeutic
medications can be used by healthy people to prevent certain infections; for example,
chloroquine to prevent malaria or isoniazid prophylaxis to prevent tuberculosis."

The search for safe and effective methods of HIV prevention continues 25 years into the HIV/AIDS epidemic. In the last decade, several biomedical prevention technologies have been tested for the prevention of sexual transmission of HIV, but only medical male circumcision has proven to be effective [1]. Trials of microbicides, herpes simplex virus-2 therapy, diaphragms and vaccines have all failed to show protection against HIV infection and, in some instances, may have even enhanced HIV infection. Much hope now rests on efficacy trials of antiretrovirals $(\mathrm{ARVs})$ as pre-exposure prophylaxis (PrEP).

ARVs have been shown to be effective in suppressing HIV replication in infected individuals [2]. However, PrEP involves the long-term use of ARVs prior to HIV exposure so that the ARVs are already biologically active in the target cells when exposure occurs, with the aim of either preventing HIV infection or, at least, favorably altering the natural course of infection to improve prognosis or decrease infectiousness. The concept of PrEP is not new; it builds on the premise that effective therapeutic medications can be used by healthy people to prevent certain infections; for example, chloroquine to prevent malaria or isoniazid prophylaxis to prevent tuberculosis. The use of ARVs as PrEP is effective in animal models; several studies since 1995 have shown that tenofovir used as PrEP can prevent SIV infection [3,4]. Even a single PrEP dose has been shown to be effective in the macaque model [5].

Further evidence to support the idea of testing ARVs for PrEP in humans comes from two sources: clinical trial data on the efficacy of ARVs in preventing mother-to-child transmission of HIV [6], and observational data showing ARVs used as postexposure prophylaxis can prevent HIV infection from needlestick exposure [7]. These create the grounds for cautious optimism as the results of efficacy trials assessing ARVs as PrEP are awaited.
To date, one trial of oral tenofovir disoproxyl fumarate $\left(\right.$ Viread $\left.^{\circledR}\right)$ involving 936 women in Ghana, Nigeria and Cameroon has been completed but was unable to address the question of effectiveness owing to the premature closure of two study sites and the low HIV incidence in the study population [8]. Other PrEP trials in Cambodia and Malawi were halted or prevented from proceeding owing to community and activist concerns $[9,10]$. Current PrEP trials underway are listed in Table 1.

Three more trials to assess topical tenofovir, Viread and oral Truvada ${ }^{\circledR}$ (oral tenofovir disoproxyl fumarate and emtricitabine) in high-risk women and discordant couples are expected to begin in 2009. The first results from the ongoing trials are expected in early 2010. If these trials demonstrate effectiveness, mathematical modeling suggests a substantial potential public health benefit; up to a $74 \%$ decline in cumulative HIV infections in 10 years from a product with $90 \%$ effective and with $75 \%$ coverage of the general population [11]. Hence, there is likely to be immense pressure for immediate implementation of PrEP if the trials are successful. However, there are several important ethical and public health issues that will need careful consideration before the widespread implementation of such an intervention.

\section{Future PrEP}

\section{implementation challenges}

Is it safe to give ARV drugs to healthy asymptomatic people?

Tenofovir has an excellent safety profile and low rates of adverse effects. Less than $1 \%$ of patients with HIV taking tenofovir in clinical trials had serious drug-related adverse events [3], although tenofovir may reduce bone density and exacerbate existing renal impairment. Emtricitabine has a similar safety profile as tenofovir and

\section{Clinical trials assessing PrEP}

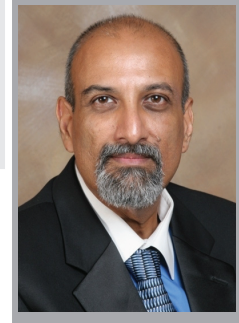

Salim S Abdool Karim Author for correspondence: CAPRISA, University of KwaZulu-Natal, Private Bag X7, Congella 4013, KwaZulu-Natal, South Africa

Tel.: +2731260 4550,

Fax: +27 312604549 ,

E-mail:karims1@ukzn.ac.za

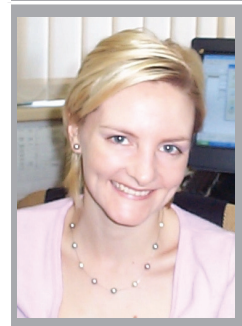

Cheryl Baxter

CAPRISA, University

of KwaZulu-Natal,

KwaZulu-Natal, South Africa 


\section{Table 1. Antiretroviral pre-exposure prophylaxis trials currently underway.}

\begin{tabular}{|c|c|c|c|}
\hline Trial & Product & Target population & Sites \\
\hline Bangkok tenofovir study & Viread $^{\circledR \ddagger}$ & 2000 intravenous drug users & Thailand \\
\hline CAPRISA 004 tenofovir gel trial & Tenofovir gel & 1000 women & South Africa \\
\hline $\begin{array}{l}\text { Botswana TDF/FTC Oral HIV } \\
\text { Prophylaxis Trial }\end{array}$ & Truvada $^{\circledR^{*}}$ & 1200 men \& women & Botswana \\
\hline iPREX trial & Truvada ${ }^{{ }^{*}}$ & 3000 men who have sex with men & $\begin{array}{l}\text { Peru, Ecuador, USA, South Africa } \\
\text { and Brazil }\end{array}$ \\
\hline PartnersPrEP trial & Viread $^{\circledR}$ and Truvada ${ }^{\circledR *}$ & 3900 discordant couples & Kenya, Uganda \\
\hline
\end{tabular}

adverse events occurring in clinical trials were generally of mild or moderate severity [12]. While mild side effects are readily tolerated when medication is taken for therapeutic reasons, the same is not necessarily true when medication is taken by healthy asymptomatic individuals, where even mild side effects may compromise adherence. Ongoing drug safety surveillance will need to be a component of plans for largescale rollout of ARVs for PrEP.

\section{Will people adhere to tablets or topical formulations for long periods?}

Long-term adherence to PrEP will be necessary for its success. Concerns about adherence to ARV therapy, especially in Africa, which emanated from preconceived notions about low education and literacy levels and knowledge of time-keeping, have repeatedly been shown to be unfounded. Studies in several African countries have convincingly demonstrated that high levels of adherence to ARVs in AIDS patients is possible [13-15]. Although this is encouraging, this may not be readily applicable to adherence in healthy asymptomatic people. While clinical trials may achieve high adherence, the same may not pertain to 'real-world' settings where PrEP would be implemented in underdeveloped public healthcare facilities without adequate attention to adherence support. On the other hand, adherence may not be an issue, as the product's effectiveness serves as strong motivation for adherence. Women have a precedent for daily pills for protection against pregnancy; however, several parts of Africa have had high pregnancy rates among women purportedly taking oral contraceptives and have switched as a result to injectables. Poor adherence may lead not only to suboptimal protection but may also impact on drug resistance. PrEP programs will need to include practical and proven adherence support programs.
Will those who get infected have HIV that is resistant to the PrEP antiretrovirals?

PrEP is proposed for use by HIV-uninfected persons, and resistance cannot develop in people who do not have the virus. However, drug resistance could possibly develop if the person taking the prophylactic regimen becomes infected with HIV while continuing to take the drugs. In the Ghana trial, no tenofovir-related resistance mutations were found in one seroconvertor in the tenofovir arm [8]. In a Phase II trial of $1 \%$ tenofovir gel (HPTN 050), no new resistance mutations were detected in plasma and cervicovaginal lavage after 14 days of product use in HIV-infected women [16].

Ultimately, the best way to prevent drug resistance in HIV is to prevent infection entirely, which is the aim of PrEP; high efficacy levels and high adherence will be important to reducing the risk of resistance. Furthermore, it is not known if the development of viral resistance mutations in persons on PrEP may lead less fit viruses, which may be rapidly replaced by wild virus, and therefore the future ARV treatment options are difficult to predict.

"...there is likely to be immense pressure for immediate implementation of PrEP if the trials are successful.

However, there are several important ethical and public health issues that will need careful consideration..."

A separate concern about resistance is the use of the same drugs (e.g., tenofovir) in therapy and prevention. Therapy failure is associated with the development of resistance and thereby the spread of resistant viruses, which in turn may compromise the efficacy of the same drugs (or, occasionally, the same class of drugs) used for prophylaxis. If PrEP is shown to be effective, 
serious consideration will need to be given to setting aside a class (or classes) of ARVs for use in prevention only. The current trials are unlikely to be able to reliably assess the full extent of the problem of drug resistance, as several hundred infections will be needed in the active product arm for this purpose.

\section{Will there be behavioral}

\section{disinhibition/risk compensation?}

A common concern when introducing new prevention technologies is whether people will stop using a more efficacious intervention for a less efficacious one. This concern is also pertinent to PrEP [17]. Mathematical modeling based on HIV epidemics in Botswana, Kenya and southern India suggests that the impact of PrEP may be diminished or even reversed by behavioral disinhibition, especially in scenarios with low coverage and low effectiveness [18]. Reassuringly, the results from the Ghana PrEP study showed that sexual risk behavior did not increase during the trial and the number of sexual partners and rate of unprotected sex acts actually decreased, probably owing to the counseling received as part of the trial [19]. Careful attention will need to be paid to messaging if PrEP is implemented to ensure existing risk-reduction behavior messaging is not undermined.

\section{"If PrEP is shown to be safe and effective, implementation programs will require substantial resources with extensive community education about the indications, availability and effectiveness of the intervention..."}

\section{Is this an affordable \& practical HIV prevention strategy for scale-up?}

Current HIV prevention programs are substantially underfunded and many highly effective prevention options such as condoms are not being used at the scale and intensity necessary to alter the epidemic trajectory [101]. Against this backdrop, the implementation of PrEP programs will have to deal with substantial logistical and financial challenges; these include HIV and hepatitis $\mathrm{B}$ virus testing, as well as renal function assessment prior to tenofovir-containing PrEP initiation and then at regular intervals of as yet unknown duration. Besides the drug costs, the programmatic and laboratory monitoring costs are likely to be substantial. One approach to reduce some of these costs is to integrate PrEP as a component of existing comprehensive HIV prevention programs and services.

\section{Conclusion}

PrEP, using ARVs, is a promising HIVprevention approach under development, and the results of current human trials are eagerly anticipated. If PrEP is shown to be safe and effective, implementation programs will require substantial resources with extensive community education about the indications, availability and effectiveness of the intervention while emphasizing the concomitant use of other proven prevention strategies. The provision of PrEP will require integration into existing HIVprevention services, which currently need to be strengthened, especially in Africa, where the need is greatest. Furthermore, there will be a need to include long-term follow-up and surveillance in sentinel groups to monitor adverse events, adherence, drug resistance, impact of drug resistance on later AIDS treatment and behavioral disinhibition.

\section{Future questions}

- Which formulation (oral or topical) of ARVs is better in terms of efficacy and safety?

- Which dosing (daily vs intermittent vs coitally related) strategy is better in terms of adherence, efficacy and safety?

- Do long-acting formulations such as vaginal rings or slow-release bolus dosing impact on adherence and risk of drug resistance?

- Are combinations of ARVs better in terms of safety, efficacy and drug resistance?

- Should a particular ARV or class of ARVs be set aside for PrEP?

\section{Financial disclosure}

The authors are conducting the CAPRISA 004 trial of tenofovir gel. CAPRISA was established through a CIPRA grant from the NIH (grant no. U19AI51794) as a multi-institutional collaboration, incorporated as an independent nonprofit AIDS Research Organization. The authors have no other relevant affiliations or financial involvement with any organization or entity with a financial interest in or financial conflict with the subject matter or materials discussed in the manuscript apart from those disclosed.

No writing assistance was utilized in the production of this manuscript. 


\section{Bibliography}

1 Auvert B, Taljaard D, Lagarde E, Sobngwi-Tambekou J, Sitta R, Puren A: Randomized, controlled intervention trial of male circumcision for reduction of HIV infection risk: the ANRS 1265 Trial. PLoS Med. 2, e298 (2005).

2 Gulick RM, Mellors JW, Havlir D et al: Treatment with indinavir, zidovudine, and lamivudine in adults with human immunodeficiency virus infection and prior antiretroviral therapy. N. Engl. J. Med. 337, 734-739 (1997).

3 Gilead Sciences Inc., Investigators Brochure: Tenofovir Gel (GS-1278) (2nd Edition). Gilead Sciences Inc., Foster City, CA, USA (2005).

4 Tsai CC, Follis KE, Sabo A et al.: Prevention of SIV infection in macaques by (R)-9(2-phosphonylmethoxypropyl)adenine. Science 270, 1197-1199 (1995).

5 Parikh UM, Sharma S, Cong ME et al.: Complete protection against repeated vaginal SHIV exposures in macaques by a combination emtricitabine and tenofovir topical gel. Presented at: XVII International AIDS Conference. Mexico, 3-8 August 2008.

6 Connor EM, Sperling RS, Gelber R et al.: Reduction of maternal-infant transmission of human immunodeficiency virus type 1 with zidovudine treatment. Pediatric AIDS Clinical Trials Group Protocol 076 Study Group. N. Engl. J. Med. 331, 1173-1180 (1994).
7 Cardo DM, Culver DH, Ciesielski CA et al: A case-control study of HIV seroconversion in health care workers after percutaneous exposure. Centers for Disease Control and Prevention Needlestick Surveillance Group. N. Engl. J. Med. 337, 1485-1490 (1997).

8 Peterson L, Taylor D, Roddy R et al.: Tenofovir disoproxil fumarate for prevention of HIV infection in women: a Phase 2, double-blind, randomized, placebo-controlled trial. PLoS Clin. Trials 2, e27 (2007).

9 Mills E: Tenofovir trials raise ethical issues. HIV AIDS Policy Law Rev. 10, 31-32 (2005).

10 Singh JA, Mills EJ: The abandoned trials of pre-exposure prophylaxis for HIV: what went wrong? PLoS Med. 2, e234 (2005).

11 Abbas UL, Anderson RM, Mellors JW: Potential impact of antiretroviral chemoprophylaxis on HIV-1 transmission in resource-limited settings. PLoS ONE 2, e875 (2007).

12 Package insert. Emtriva, Gilead Sciences Inc., CA, USA (2003).

13 van Oosterhout JJ, Bodasing N, Kumwenda JJ et al.: Evaluation of antiretroviral therapy results in a resource-poor setting in Blantyre, Malawi. Trop. Med. Int. Health 10, 464-470 (2005).

14 Nachega JB, Stein DM, Lehman DA et al.: Adherence to antiretroviral therapy in HIV-infected adults in Soweto, South Africa. AIDS Res. Hum. Retroviruses 20, 1053-1056 (2004).
15 Byakika-Tusiime J, Oyugi JH, Tumwikirize WA et al:: Adherence to HIV antiretroviral therapy in $\mathrm{HIV}^{+}$Ugandan patients purchasing therapy Int. J. STD AIDS 16, 38-41 (2005).

16 Mayer KH, Maslankowski LA, Gai F et al.: Safety and tolerability of tenofovir vaginal gel in abstinent and sexually active HIV-infected and uninfected women. AIDS 20, 543-551 (2006).

17 Liu AY, Grant RM, Buchbinder SP: Preexposure prophylaxis for HIV: unproven promise and potential pitfalls. JAMA 296, 863-865 (2006).

18 Vissers DC, Voeten HA, Nagelkerke NJ, Habbema JD, de Vlas SJ: The impact of pre-exposure prophylaxis (PrEP) on HIV epidemics in Africa and India: a simulation study. PLoS ONE 3, e2077 (2008).

19 Guest G, Shattuck D, Johnson L et al.: Changes in sexual risk behavior among participants in a PrEP HIV prevention trial. Sex. Transm. Dis. (2008) (Epub ahead of print).

\section{Website}

101 WHO. Coverage of selected health services for HIV/AIDS prevention and care in less developed countries in 2001. WHO, Geneva, Switzerland (2002). http://whqlibdoc.who.int/publications/ 9241590319.pdf 